\section{Absent CNKSR2 Causes Seizures and Intellectual, Attention, and Language Deficits}

\author{
Andrea K. Vaags, PhD, 1,2,3 \\ Sarah Bowdin, BM, MSc, MRCPCH, ${ }^{4,5}$ \\ Mary-Lou Smith, PhD, 6,7 \\ Brigitte Gilbert-Dussardier, MD, ${ }^{8}$ \\ Katja S. Brocke-Holmefjord, MD, ${ }^{9}$ \\ Katia Sinopoli, PhD, CPsych, 10,11 \\ Cindy Gilles, $\mathrm{MSc}^{12}$ \\ Tove B. Haaland, MD, ${ }^{9}$ \\ Catherine Vincent-Delorme, MD, ${ }^{13}$ \\ Emmanuelle Lagrue, MD, ${ }^{14}$ \\ Radu Harbuz, MD, 8 \\ Susan Walker, PhD, 1,15 \\ Christian R. Marshall, PhD, 1,15,16,17 \\ Gunnar Houge, MD, PhD, ${ }^{18}$ \\ Vera M. Kalscheuer, PhD, ${ }^{19}$ \\ Stephen W. Scherer, PhD, 1,15,16,17 and \\ Berge A. Minassian, MD 6,15,20,21
}

Synaptic function is central to brain function. Understanding the synapse is aided by studies of patients lacking individual synaptic proteins. Common neurological diseases are genetically complex. Their understanding is likewise simplified by studies of less common monogenic forms. We detail the disease caused by absence of the synaptic protein CNKSR2 in 8 patients ranging from 6 to 62 years old. The disease is characterized by intellectual disability, attention problems, and abrupt lifelong language loss following a brief early childhood epilepsy with continuous spike-waves in sleep. This study describes the phenotype of CNKSR2 deficiency and its involvement in systems underlying common neurological disorders.

ANN NEUROL 2014;76:758-764

ntellectual disability, attention-deficit/hyperactivity disorder (ADHD), epilepsy, and developmental language impediments are common genetically complex disorders. Cases caused by single gene defects are invaluable in elucidating pathogenic processes, which might be shared with the common forms of these diseases.

The postsynaptic density (PSD) consists of $\sim 1,400$ diffusible and structural proteins. The latter are organized into 3 layers: a membrane-anchored layer (cell-adhesion, ion-channel, and receptor proteins), a deep layer (SHANK and guanylate-kinase-associated proteins), and between the two a large intermediate layer of numerous scaffold and adaptor proteins, including CNKSR2. ${ }^{1,2}$ CNKSR2 physically links with the major PSD proteins densin-180, PSD95, and S-SCAM and regulates Ras signaling, which controls neuronal proliferation, migration, differentiation, and death, ${ }^{3-7}$ as well as Ras-mediated synaptogenesis. ${ }^{8,9}$ A first report of a patient lacking CNKSR2 was recently published. The patient had developmental delay, well-controlled epilepsy, and microcephaly. ${ }^{10}$ We now present comprehensive descriptions of 8 patients with this defect, including the original case (Patient 3). This work establishes the neurodevelopmental and neurological syndrome of absent CNKSR2.

\section{Patients and Methods}

The study was approved by the Hospital for Sick Children research ethics board. Informed consent was obtained from

From the ${ }^{1}$ Centre for Applied Genomics, Hospital for Sick Children, Toronto, Ontario, Canada; ${ }^{2}$ Cytogenetics Laboratory, Alberta Children's Hospital, Calgary, Alberta, Canada; ${ }^{3}$ Department of Anatomical Pathology and Cytopathology, Calgary Laboratory Services, Calgary, Alberta, Canada; ${ }^{4}$ Division of Clinical and Metabolic Genetics, Hospital for Sick Children, Toronto, Ontario, Canada; ${ }^{5}$ Division of Paediatrics, Faculty of Medicine, University of Toronto, Toronto, Ontario, Canada; ${ }^{6}$ Neurosciences and Mental Health Program, Hospital for Sick Children, Toronto, Ontario, Canada; ${ }^{7}$ Department of Psychology, University of Toronto, Toronto, Ontario, Canada; ${ }^{8}$ Genetic Service, Poitiers University Hospital Centre, University of Poitiers, Poitiers, France; ${ }^{9}$ Department of Pediatric Habilitation, Stavanger University Hospital, Stavanger, Norway; ${ }^{10}$ Division of Neurology, Hospital for Sick Children, Toronto, Ontario, Canada; ${ }^{11}$ Department of Psychology, Hospital for Sick Children, Toronto, Ontario, Canada; ${ }^{12}$ Pediatric Neurology Unit, Gatien de Clocheville Children's Hospital, Tours Regional University Hospital Centre, Tours, France; ${ }^{13}$ Guy Fontaine Clinical Genetics Service, Jeanne de Flandre Hospital, Lille, France; ${ }^{14}$ Children's Neuromuscular Consultation, Neuropediatric and Handicap Service, Gatien de Clocheville Children's Hospital, Tours Regional University Hospital Centre, Tours, France; ${ }^{15}$ Program in Genetics and Genome Biology, Hospital for Sick Children, Toronto, Ontario, Canada; ${ }^{16}$ Department of Molecular Genetics, University of Toronto, Toronto, Ontario, Canada; ${ }^{17}$ McLaughlin Centre, University of Toronto, Toronto, Ontario, Canada; ${ }^{18}$ Center for Medical Genetics and Molecular Medicine, Haukeland University Hospital, Bergen, Norway; ${ }^{19}$ Department of Human Molecular Genetics, Max Planck Institute for Molecular Genetics, Berlin, Germany; ${ }^{20}$ Division of Neurology, Faculty of Medicine, University of Toronto, Toronto, Ontario, Canada; and ${ }^{21}$ Institute of Medical Sciences, University of Toronto, Toronto, Ontario, Canada.

Address correspondence to Berge A. Minassian, MD, Room 6536B, The Hospital for Sick Children 555 University Ave. Toronto, Ontario M5G 1X8, Canada Tel: 416-813-6291; Fax: 416-813-6334

E-mail: berge.minassian@sickkids.ca

Additional Supporting Information may be found in the online version of this article.

Received Jun 12, 2014, and in revised form Sep 11, 2014. Accepted for publication Sep 11, 2014.

View this article online at wileyonlinelibrary.com. DOI: 10.1002/ana. 24274 
A

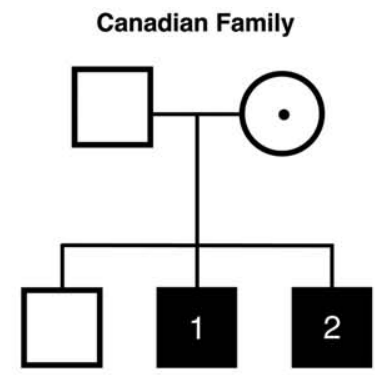

Norwegian Family

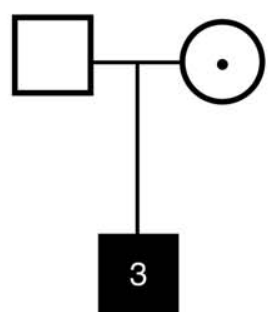

French Family 1

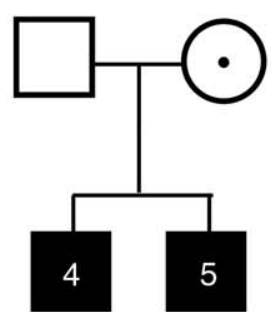

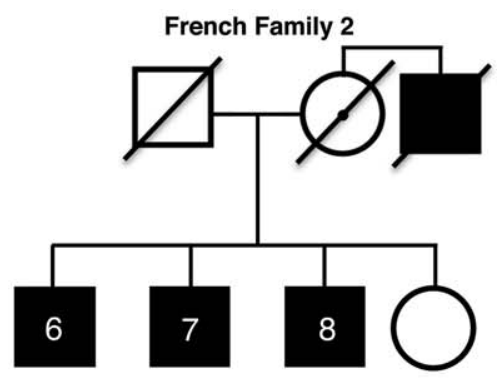

B

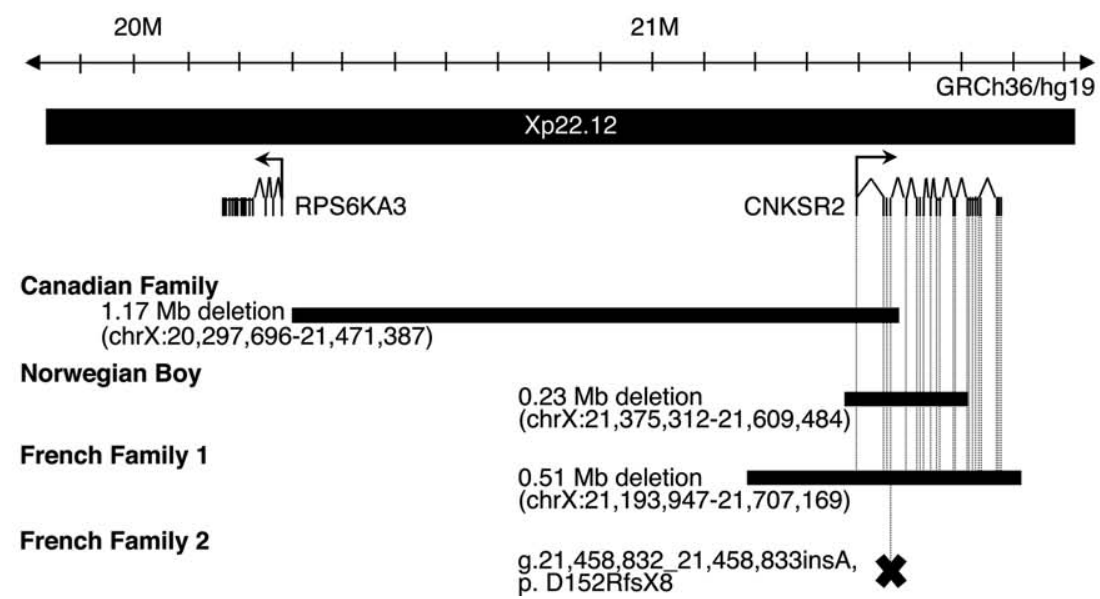

FIGURE 1: Genetic details. (A) Pedigrees of the 4 families; solid squares = affected males; open circles with black dots $=$ carrier mothers. (B) Genomic map of chromosome Xp22.12 with extent of deletions (black bars) or position of frameshift premature termination codon (black X). The deletion in the Canadian family approximated the RPS6KA3 gene, deletions of which cause the Coffin-Lowry syndrome, which our patients do not have. However, fine-mapping the breakpoints of the 1.17Mb deletion showed that it does not involve this gene, and Western blot analysis confirmed that expression of the RPS6KA3 protein product, RSK2, is unaltered (data not shown).

parents or caregivers. Five boys, aged 13 years and younger, with developmental delay, language deficits, seizures (in 4), and unremarkable magnetic resonance imaging (MRI) were tested for copy number variation $(\mathrm{CNV})$ and found to have deletions of the X-linked CNKSR2 gene (Fig 1). Examination of the Database of Genomic Variants revealed no $\mathrm{CNV}$ of CNKSR2 in healthy control individuals. ${ }^{11}$ We describe their neurological, neurodevelopmental, and neuropsychological features, the latter with standardized psychometric testing adapted to culture and language (Supplementary Table). We also describe 3 brothers, aged 56 years and older, who share a frameshift-premature termination mutation in CNKSR2 (see Fig 1) identified through a multinational intellectual disability X-chromosome exome sequencing project (in preparation).

\section{Results}

The Table 1 summarizes the genetic and clinical features of all 8 patients. The Supplementary Table details the psychometric testing of all 8 patients and the parents of Patients 1 and 2.
Patient 1, a 6-year-old boy, walked at age 2 years and spoke multiple words by that time. He had a generalized nonfebrile sleep-related seizure soon after he walked and lost most of his words. Overnight electroencephalogram (EEG) showed frontotemporal-dominant continuous spike-and-slow-waves (CSWS; Fig 2A). Valproic acid prevented further seizures. At age 3 years 6 months, testing showed poor attention, hyperactivity, and intellectual, especially language delay (verbal intelligence quotient $[\mathrm{IQ}] \leq 3$ rd percentile; receptive vocabulary $\leq 5$ th percentile). At age 4 years, his CSWS were treated with high-dose diazepam. Baseline overnight EEG showed CSWS at 80 to $100 \%$ of non-rapid eye movement (REM) sleep. Diazepam $(0.5 \mathrm{mg} / \mathrm{kg})$ was administered intravenously at 8 and $9 \mathrm{PM}$, and on the subsequent night CSWS were reduced to 0 to $50 \%$ of non-REM sleep. An overnight EEG 3 months later showed CSWS at 50 to $75 \%$. With no improvement in language, at 4 years 10 months he was treated with prednisone, $40 \mathrm{mg}$ daily over 6 weeks followed by a 7-week weaning. Repeat 


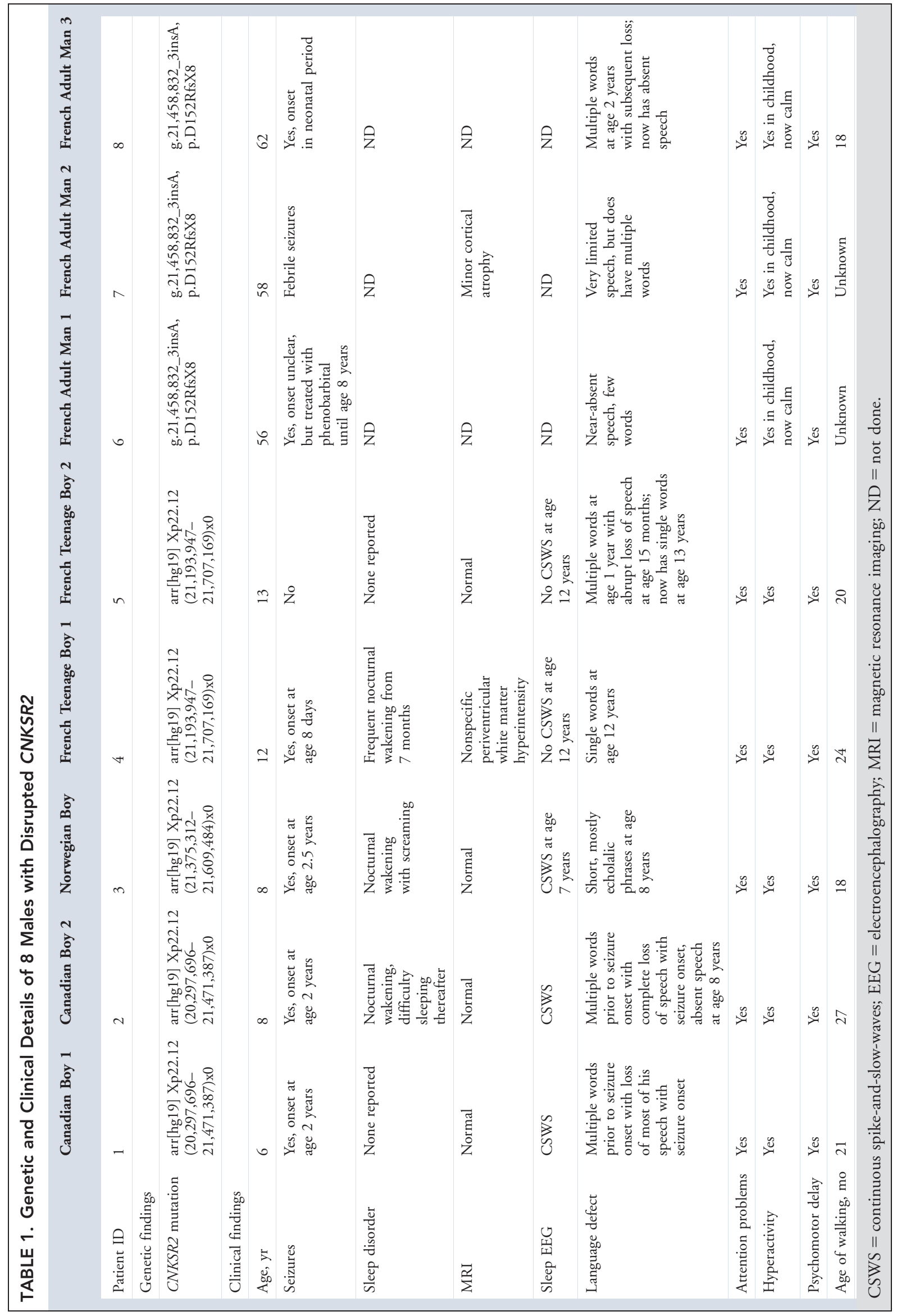


A

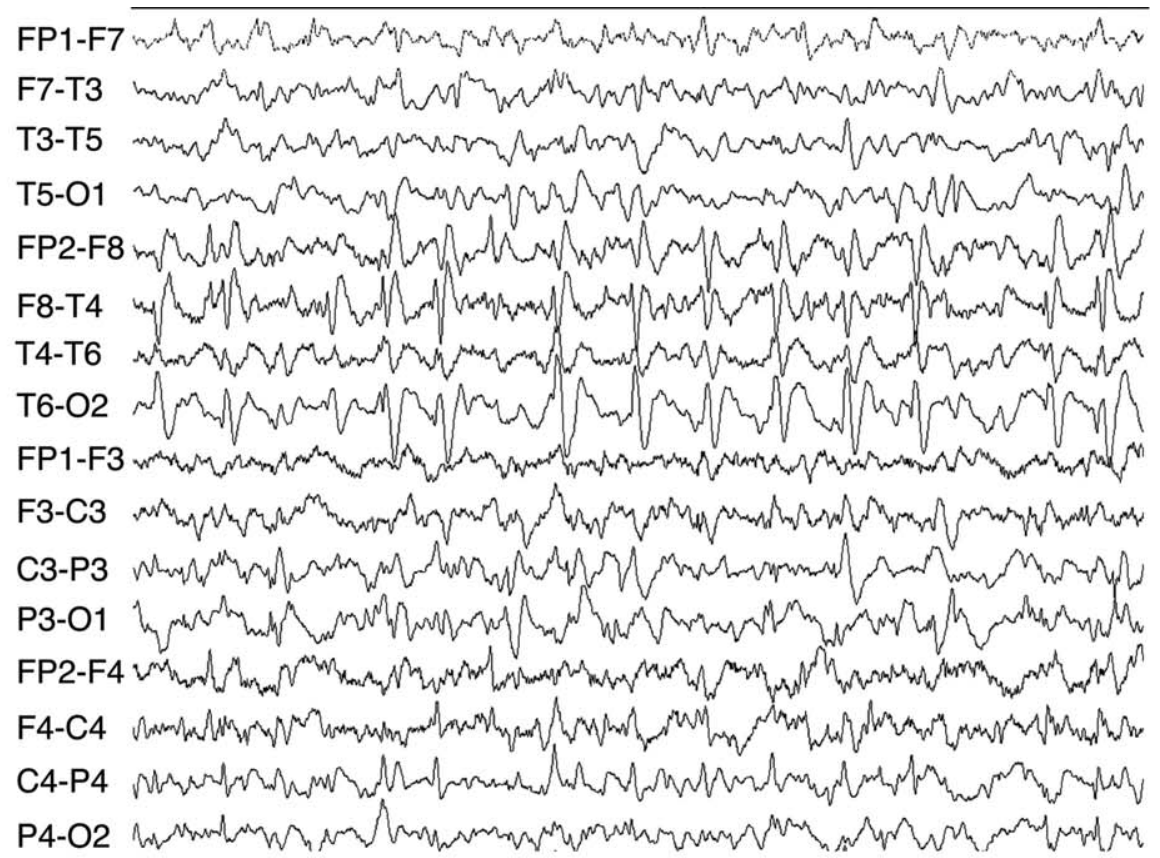

B

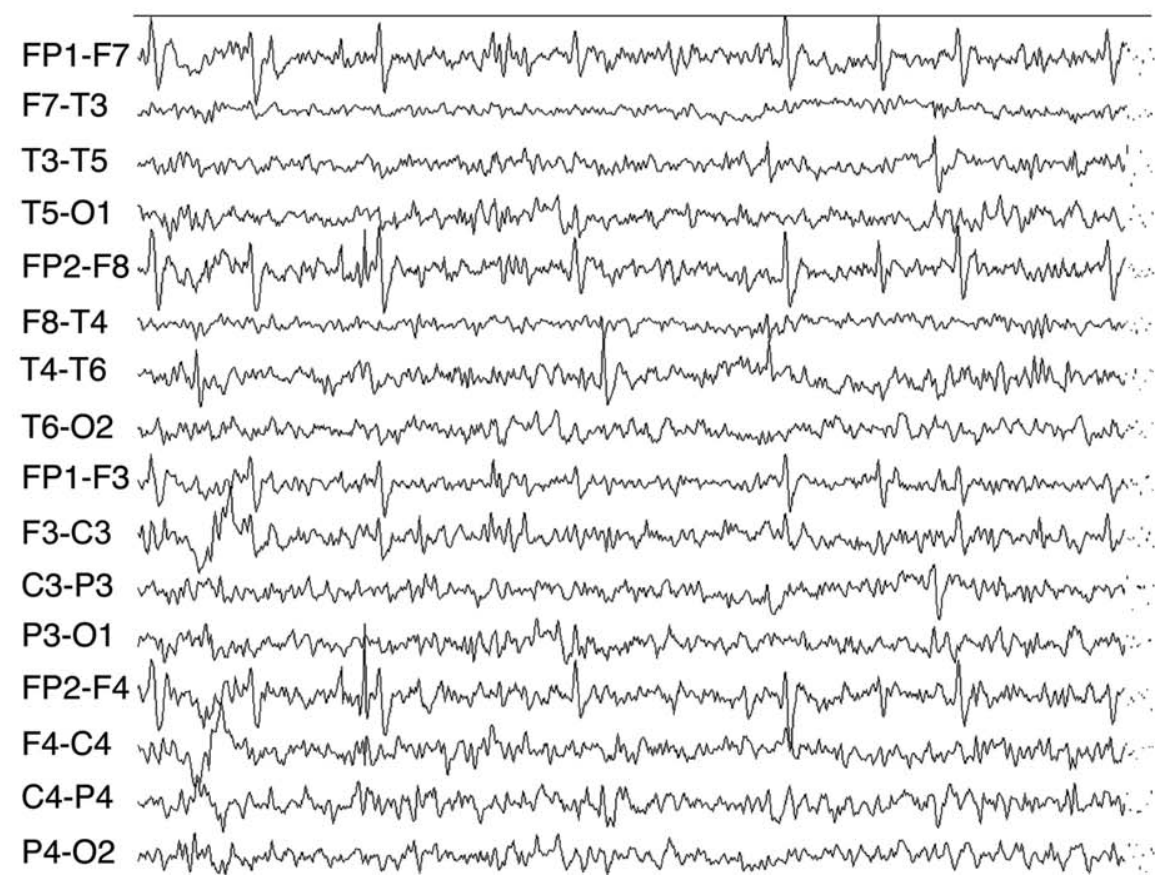

FIGURE 2: Sleep electroencephalogram (EEG) longitudinal bipolar montage. (A) Sample from Patient 1 (sleep stage N2). Throughout non-rapid eye movement sleep continuous spike-and-slow-waves were mostly symmetrically bifrontotemporal, although they were sometimes unilateral dominant, in either hemisphere, as in the right-sided example shown here. Wakeful EEG had normal posterior alpha rhythm, with infrequent symmetric or independent frontotemporal spikes. (B) Sleep EEG sample from Patient 3 (early stage N1 sleep). Wakeful EEG showed infrequent symmetric or independent frontally dominant spikes.

EEG showed CSWS occupying approximately $90 \%$ of non-REM sleep. Neuropsychological assessment at age 5 years indicated a lack of developmental gains, with major delays in intellectual function ( $\leq 1$ st percentile), including language function (verbal IQ $\leq 0.1$ percentile; receptive vocabulary $\leq 1$ st percentile).
Patient 2 is the 8 -year-old brother of Patient 1 . $\mathrm{He}$ walked at 2 years, having acquired 10 words. At 27 months, he experienced a prolonged nonfebrile sleep-related generalized tonic-clonic seizure, and stopped speaking. He had several self-limited generalized nonfebrile seizures on carbamazepine. Overnight video- 
EEG at age 4 years showed rhythmic frontotemporaldominant CSWS exceeding $80 \%$ of the record during non-REM sleep. On valproic acid, he had no further seizures. Neuropsychological testing showed global developmental delay with no language, impaired attention, and hyperactivity.

The mother, a carrier of the deletion, had mild learning disability in childhood affecting reading and mathematics. Neuropsychological assessment at age 41 years indicated normal overall intellectual function, with a deficit in completing numerical operations. Her overnight EEG was normal, as was that of her unaffected third son, who did not have the CNKSR2 deletion, and the father of the 3 boys.

Patient 3, 8 years old, babbled in the first year of life and walked at 18 months. At 2 years 6 months, he developed staring spells and a single generalized tonicclonic seizure, controlled by lamotrigine. At age 6 years, he had single words or echolalic phrases (eg, when asked, "Do you go to kindergarten?" he would not respond, but repeat, "Go kindergarten."). He was diagnosed with ADHD and treated with methylphenidate. Sleep EEG at age 7 years showed CSWS (see Fig 2B), and neuropsychological assessment revealed significant intellectual disability ( $\leq 1$ st percentile), inattention, and impulsivity.

Patient 4, 12 years old, was born at 31 weeks gestational age with no etiology determined for his prematurity. He required treatment for hyaline membrane disease, developing seizures on day 8 of life with right-sided hemiparesis. The latter resolved, and he now has a normal neurological examination. He had several complex partial and generalized seizures, controlled with carbamazepine and valproic acid. He has frequent nocturnal awakenings and severe hyperactivity, treated with the dopamine antagonist tiapride. MRI showed nonspecific bilateral periventricular hyperintensities. EEGs during wakefulness showed active spiking from the left temporal lobe. Overnight EEG at age 12 years did not reveal CSWS. Formal psychometric assessment confirmed major intellectual disability and inattention.

Patient 5, 13 years old, is the brother of Patient 4 . He walked at 20 months. He had cessation of language development at 15 months, and has attention problems, hyperactivity, and aggressive behavior, treated with methylphenidate. He has had no seizures. Overnight EEG at age 12 years revealed no CSWS but showed infrequent left hemispheric and generalized spike-waves. Formal psychometric assessment confirmed major intellectual disability, with marked improvement in attention and behavior.

Patients 6 to 8 , aged 56, 58, and 62 years, live in an institution and have either no or limited speech. Data on these patients' childhood was obtained in large part from their lifelong caregiver sister. The eldest spoke in short phrases in early childhood, which he then lost. Early speech histories in the other brothers were unavailable. All had major attention deficit and hyperactivity throughout childhood, but are presently calm. Febrile seizures were present in childhood in the middle brother and afebrile seizures in the other two. There have been no seizures beyond age 10 years in any, and none is presently on antiepileptic medications. The middle brother had a recent MRI, which showed minor cortical atrophy.

\section{Discussion}

The cardinal features of CNKSR2 deficiency are: (1) intellectual disability, (2) highly restricted speech (especially expressive language), (3) attentional problems/ hyperactivity, and (4) brief childhood epilepsy. A fifth feature may be CSWS in early childhood. Of 8 patients studied, 3 who were younger than 10 years (from 2 separate families) had CSWS, whereas the 5 who were older did not. CSWS in encephalopathy related to electrical status epilepticus during sleep (ESES) and in LandauKleffner syndrome (LKS) are developmental stage limited and disappear in the teenage years. ${ }^{12}$ Whether this is what we are observing in the spread of ages in our set of patients remains to be determined with future cases and with follow-up of the 3 youngest boys. If it is, one would ask whether CSWS are contributing to the developmental and language disorder in this disease, as it does in ESES and LKS. ${ }^{12}$ It is notable that half our patients had a clear history of some language prior to their first seizures, which they then lost and never reacquired. The CSWS in the 3 young children were resistant to standard antiepileptic management, and also to aggressive therapies with high-dose diazepam and corticosteroids, as they are in most cases of ESES and LKS. ${ }^{12}$ The seizures themselves readily responded to standard therapy, reminiscent of most cases of ESES and LKS. ${ }^{12}$

Our patients had developmental delay prior to the onset of seizures. This is consistent with the finding that CNKSR2 is expressed prenatally, suggesting a role in neurodevelopment. In the adult brain, it is expressed ubiquitously, indicating a general synaptic or synaptogenic function. ${ }^{13,14}$

LKS and ESES, with benign rolandic epilepsy, are a continuum of related diseases, sharing sleep-enhanced epileptic discharges and associated behavioral and cognitive deficits ${ }^{15}$ and male predominance. ${ }^{15,16}$ Gene mutations are considered to play an important, albeit complex, role in this spectrum of diseases. Currently, one genetic cause is known (Online Mendelian Inheritance in Man database [OMIM] 138253, GRIN2A, encoding the 
N2RA subunit of the N-methyl-D-aspartate receptor), explaining a fraction of these patients. Whether CNKSR2 mutations, milder than the deleting mutations reported here, might be additional causes awaits future studies.

ADHD is among the most heritable neuropsychiatric diseases, and is genetically complex. ${ }^{17}$ ADHD is a common feature of many single gene disorders including tuberous sclerosis, fragile $\mathrm{X}$ syndrome, and neurofibromatosis type 1 , as well as microdeletion syndromes such as Smith-Magenis syndrome and 22q11.2 deletion syndrome. ${ }^{18}$ Combinations of genome-wide association and CNV studies have revealed a small portion of the disorder's gene risk. ${ }^{19,20}$ ADHD is also 4-fold more common in boys than girls, ${ }^{21}$ suggesting substantial contributions from X-chromosome loci. However, genome-wide association studies, for methodological reasons, have not considered the $\mathrm{X}$ chromosome. ${ }^{22}$ The 8 males lacking CNKSR2 due to CNV or nonsense mutations have prominent attentional problems/hyperactivity. Whether milder CNKSR2 mutations might be found in other ADHD cohorts remains to be seen.

CNKSR2 joins the growing list of genes encoding PSD proteins, which, when mutated, cause a broad range of neurodevelopmental disorders. Notable examples include SHANK3 (OMIM 606230), which is associated with autism, schizophrenia, and intellectual disability, and DLG3 (OMIM 300189), which is associated with intellectual disability. Further delineation of the molecular pathogenesis of this group of conditions will allow the development of individualized treatments on the basis of molecular diagnosis, replacing current empiric approaches.

\section{Acknowledgment}

This work was supported by grants from the Government of Ontario, Canadian Institute for Advanced Research, McLaughlin Foundation, Ontario Brain Institute, and Genome Canada to S.W.S. and B.A.M., and by the French Health Ministry to B.G.-D., and R.H.

B.A.M. holds the University of Toronto Michael Bahen Chair in Epilepsy Research. S.W.S. holds the GlaxoSmithKline-Canadian Institutes for Health Research Chair in Genome Sciences at the University of Toronto and the Hospital for Sick Children. A.K.V. is the recipient of a Calgary Laboratory Services Cytogenetics Fellowship.

We thank clinicians M. Zak, D. Molinari Andrade, W. Roberts, P. Cooper, and F. Bilan for the care of these patients and J. Stavropoulos, P. Wang, A. Lionel, and B. Thiruvahindrapuram for experimental contributions.

\section{Authorship}

A.K.V. and S.B. contributed equally to this work.

\section{Potential Conflicts of Interest}

Nothing to report.

\section{References}

1. Bayes A, van de Lagemaat LN, Collins $M O$, et al. Characterization of the proteome, diseases and evolution of the human postsynaptic density. Nat Neurosci 2011;14:19-21.

2. Feng $W$, Zhang M. Organization and dynamics of PDZ-domainrelated supramodules in the postsynaptic density. Nat Rev Neurosci 2009;10:87-99.

3. Bumeister R, Rosse C, Anselmo A, et al. CNK2 couples NGF sig nal propagation to multiple regulatory cascades driving cell differentiation. Curr Biol 2004;14:439-445.

4. Liu L, Channavajhala PL, Rao VR, et al. Proteomic characterization of the dynamic KSR-2 interactome, a signaling scaffold complex in MAPK pathway. Biochim Biophys Acta 2009;1794:1485-1495.

5. Ohtakara K, Nishizawa M, Izawa I, et al. Densin-180, a synaptic protein, links to PSD-95 through its direct interaction with MAGUIN-1. Genes Cells 2002;7:1149-1160.

6. Rajakulendran T, Sahmi M, Kurinov I, et al. CNK and HYP form a discrete dimer by their SAM domains to mediate RAF kinase signaling. Proc Natl Acad Sci U S A 2008;105:2836-2841.

7. Yao I, Ohtsuka T, Kawabe $\mathrm{H}$, et al. Association of membraneassociated guanylate kinase-interacting protein-1 with Raf-1. Biochem Biophys Res Commun 2000;270:538-542.

8. Lanigan TM, Liu A, Huang YZ, et al. Human homologue of Drosophila CNK interacts with Ras effector proteins Raf and Rlf. FASEB J 2003;17:2048-2060.

9. Hotulainen $\mathrm{P}$, Llano $\mathrm{O}$, Smirnov $\mathrm{S}$, et al. Defining mechanisms of actin polymerization and depolymerization during dendritic spine morphogenesis. J Cell Biol 2009;185:323-339.

10. Houge G, Rasmussen IH, Hovland R. Loss-of-function CNKSR2 mutation is a likely cause of non-syndromic X-linked intellectual disability. Mol Syndromol 2012;2:60-63.

11. MacDonald JR, Ziman R, Yuen RK, et al. The Database of Genomic Variants: a curated collection of structural variation in the human genome. Nucleic Acids Res 2014;42(Database issue): D986-D992.

12. Tassinari CA, Cantalupo G, Dalla Bernardina B, et al. Encephalopathy related to status epilepticus during slow sleep (ESES) including Landau-Kleffner syndrome. In: Bureau M, Genton P, Dravet $C$, et al, eds. Epileptic syndromes in infancy, childhood and adolescence. 5th ed. London, UK: John Libbey, 2012:255275.

13. Nagase $T$, Ishikawa $K$, Miyajima $N$, et al. Prediction of the coding sequences of unidentified human genes. IX. The complete sequences of 100 new cDNA clones from brain which can code for large proteins in vitro. DNA Res 1998;5:31-39.

14. Stiffler MA, Grantcharova VP, Sevecka M, et al. Uncovering quantitative protein interaction networks for mouse PDZ domains using protein microarrays. J Am Chem Soc 2006;128: 5913-5922.

15. Tsai MH, Vears DF, Turner SJ, et al. Clinical genetic study of the epilepsy-aphasia spectrum. Epilepsia 2013;54:280-287.

16. Appleton RE. The Landau-Kleffner syndrome. Arch Dis Child $1995 ; 72: 386-387$. 
ANNALS of Neurology

17. Faraone SV, Doyle AE. The nature and heritability of attentiondeficit/hyperactivity disorder. Child Adolesc Psychiatr Clin N Am 2001;10:299-316, viii-ix.

18. Thapar A, Cooper M, Jefferies R, Stergiakouli E. What causes attention deficit hyperactivity disorder? Arch Dis Child 2012;97:260-265.

19. Elia J, Glessner JT, Wang K, et al. Genome-wide copy number variation study associates metabotropic glutamate receptor gene networks with attention deficit hyperactivity disorder. Nat Genet 2012;44:78-84.
20. Lionel AC, Crosbie J, Barbosa N, et al. Rare copy number variation discovery and cross-disorder comparisons identify risk genes for ADHD. Sci Transl Med 2011;3:95ra75.

21. Cantwell DP. Attention deficit disorder: a review of the past 10 years. J Am Acad Child Adolesc Psychiatry 1996;35:978987.

22. Wise $A L$, Gyi L, Manolio TA. eXclusion: toward integrating the $X$ chromosome in genome-wide association analyses. Am J Hum Genet 2013;92:643-647. 